\title{
Totally unordered subsets of a partially well ordered set
}

\author{
Martin Ward
}

\begin{abstract}
An order on the collection of totally unordered subsets of a partially well ordered set is defined, shown to satisfy the descending chain condition but not to be necessarily a partial well order itself.
\end{abstract}

Let $\leq$ be a partial order on a set $W$. Two elements $a$ and $b$ of $W$ are comparable if either $a \leq b$ or $b \leq a$, otherwise they are incomparable. A subset $T$ of $W$ is totally unordered if all the elements of $T$ are incomparable with each other. The partial order $\leq$ is a partial well order if every non weakly ascending sequence, that is every sequence $x_{0}, x_{1}, \ldots$ for which $i<j \Rightarrow x_{i} \neq x_{j}$, is finite or, equivalently, if it satisfies the descending chain condition and every totally unordered subset is finite.

The subsets of $W$ may be pre-ordered as follows: for subsets $A$ and $B$ of $W$, write $A \leq B$ if for all $b \in B$, there exists $a \in A$ such that $a \leq b$. The restriction of this relation to the collection of totally unordered subsets of $W$ is a partial order.

Examples of the role of partial well orders in algebra can be found in [1] and an example of the natural occurrence of the pre-order on the subsets of a partially well ordered set given above, together with its restriction to the totally unordered subsets can be found in [2]. In the

Received 24 November 1969. 
latter paper the following theorem is quite important; it appears there (as Lemma 4.1, Corollary) "proved by intimidation" but appears to deserve better treatment than this.

THEOREM 1. With the notation defined above, the order $\leq$ on the collection of totally unordered subsets of $W$ satisfies the descending chain condition.

Proof is by contradiction. Assume that

$$
T_{0}>T_{1}>T_{2}>\ldots
$$

is an infinite descending chain of totally unordered subsets of $W$. For each non-negative integer $i$ define a set $\Delta_{i}$ by

$$
\Delta_{i}=T_{i}-\underset{j<i}{U} T_{j}
$$

so that, in particular, $\Delta_{0}=T_{0}$. Then clearly the $\Delta_{i}$ are mutually disjoint. Suppose there were only a finite number of integers $i$ such that $\Delta_{i}$ is nonempty. Then there would be a largest such integer, $i_{0}$ say. But then, for all $j>i_{0}, T_{j}$ would be a subset of $\underset{i \leq i_{0}}{U} T_{i}$, which is a finite set since each $T_{i}$, being totally unordered, is finite. Then $T_{i_{0}+1}, T_{i_{0}+2}, \cdots$ would be an infinite sequence of distinct subsets of a finite set, which is impossible. Thus there exists an infinite ascending sequence $m_{0}<m_{1}<\ldots$ of non-negative integers such that $\Delta_{m_{i}}$ is nonempty for each $i$. Invoking the axiom of choice, there exists a sequence $\delta_{0}, \delta_{1}, \ldots$ in $W$ such that $\delta_{i} \in \Delta_{m_{i}}$ for each $i$. Then there exists $i$ and $j$ such that $i<j$ and

$$
\delta_{i} \leq \delta_{j}
$$

for otherwise this sequence would be non weakly ascending, contradicting the fact that $W$ is partially well ordered. But then $\delta_{i} \in \Delta_{m_{i}} \subseteq T_{m_{i}}>T_{m_{j}}$ so there exists $\tau \in T_{m_{j}}$ such that

$$
\delta_{i} \geq \tau
$$


and then, from (I) and (2), $\tau \leq \delta_{j}$. But $\tau$ and $\delta_{j}$ are both members of the totally unordered set $T_{m_{j}}$, so $\tau=\delta_{j} \cdot$ This, with (1) and (2), implies that $\delta_{i}=\delta_{j}$ contradicting the disjointness of $\Delta_{m_{i}}$ and $\Delta_{m_{j}}$.

It should be remarked that sets with partial well orders that appear in algebra usually come equipped with a full well order also. In this situation, the underlying set has a choice function and the services of the axiom of choice can be dispensed with in the proof above.

In view of the generally tractable nature of partial well orders, it might be asked if this collection of totally unordered subsets of $W$ is itself partially well ordered. That this is not necessarily so is shown by the following example. Let $W$ be the set of all pairs $(m, n)$ of integers such that $l \leq m \leq n$. Define an order $\leq$ on $W$ by

$$
\begin{gathered}
\left(m_{1}, n_{1}\right) \leq\left(m_{2}, n_{2}\right) \text { if either } n_{1} \leq n_{2} \text { and } m_{1}=m_{2} \\
\text { or } n_{1}+2 \leq m_{2} \text {. }
\end{gathered}
$$

Then $W$ is partially well ordered but the infinite collection $\left\{T_{1}, T_{2}, \ldots\right\}$ of totelly unordered sets defined by

$$
T_{n}=\{(m, n): 1 \leq m \leq n\}
$$

is itself totally unordered.

THEOREM 2. For each non-negative integer $n$, the collection of totally unordered subsets of $W$ which contain no more than $n$ elements each is partially well ordered.

Proof is by induction over $n$. For $n=0$ the result is trivial since there is only one such totally unordered set, namely the empty set. Now suppose $n \geq 1$ and the result is true for totally unordered sets which contain no more than $n-1$ elements. Let $T$ be an infinite collection of distinct totally unordered subsets of $W$ which contain no more than $n$ elements each. By virtue of Theorem $l$ it is sufficient to show that $T$ is not itself totally unordered. Since $T$ is infinite, so is its union UT which must then contain an infinite ascending sequence,

$$
t_{0}<t_{1}<\ldots
$$


say. For each non-negative integer $i$, there exists $T_{i} \in T$ such that $t_{i} \in T_{i}$. Then

$$
T_{0}-t_{0}, T_{1}-t_{1}, \ldots
$$

is an infinite sequence of totally unordered subsets of $W$ each of which contains no more than $n-1$ elements, so by the inductive hypothesis there exists $i<j$ such that $T_{i}-\left\{t_{i}\right\} \leq T_{j}-\left\{t_{j}\right\}$. But then, since $t_{i}<t_{j}$ it follows that $T_{i} \leq T_{j}$. Thus $T$ is not totally unordered.

\section{References}

[1] Graham Higman, "Ordering by divisibility in abstract algebras", Proc. London Math. Soc. (3) 2 (1952), 326-336.

[2] M.A. Ward, "Basic commutators", Phizos. Trans. Roy. Soc. London Ser. A 264 (1969), 343-412.

Australian National University,

Canberra, ACT. 